\title{
Avaliação do aumento da estabilidade primária na instalação de implantes dentários com variação da instrumentação por meio do torque de inserção e análise de frequência de ressonância
}

\author{
- Maurício Makoto Koga Discipline of Implantology, School of Dentistry, São Leopoldo Mandic University, Campinas, SP, Brazil \\ - Alexander D Salvoni Discipline of Implantology, School of Dentistry, São Leopoldo Mandic University, Campinas, SP, Brazil \\ - Thomaz Wassall Discipline of Implantology, School of Dentistry, São Leopoldo Mandic University, Campinas, SP, Brazil
}

RESUMO | Introdução: A descoberta da osseointegração determinou um marco para a terapia reabilitadora na odontologia, possibilitando tratamentos previsíveis. Objetivo: O objetivo deste estudo foi avaliar uma técnica alternativa de instalação de implantes que proporcionasse melhor estabilidade primária. Materiais e métodos: Foram selecionadas seis costelas suínas frescas para a instalação de 60 implantes cilíndricos usinados divididos em três grupos. Anteriormente à instalação dos implantes, foi realizada a medição da densidade óssea em unidade de Hounsfield com tomografia computadorizada convencional. Os valores de densidade óssea foram medidos na posição dos implantes e correlacionados ao torque de inserção e à análise de frequência de ressonância. No grupo-controle usou-se a técnica de instalação onde a última broca utilizada foi a de 3,o mm preconizada pelo fabricante, enquanto nos grupos 2 e 3 foi utilizada uma técnica alternativa de subinstrumentação e superinstrumentação, onde as últimas brocas utilizadas no preparo do leito cirúrgico foram as de $2,8 \mathrm{~mm}$ e 3,15 mm, respectivamente. Foram registrados os valores de torque de inserção máxima no momento da instalação dos implantes, e os valores da frequência de ressonância foram medidos a partir do quociente de estabilidade implantar (ISQ). Resultados: Foi registrado aumento do torque de inserção de 63,1219\% quando se utilizou a broca de 2,8 mm como broca final, e se obteve um torque negativo de inserção na marca de -4,071\% quando se utilizou a broca 3,15 mm como broca final, na comparação com o grupocontrole. Foi verificada uma correlação entre a estabilidade primária, obtida através da média do torque de inserção do implante, e o diâmetro de preparo do leito receptor do implante com a utilização do implante cilíndrico. Conclusão: Os resultados indicam que utilizando essa técnica de preparo alternativo de subinstrumentação o cirurgião pode atingir valores de torque de inserção mais elevados mesmo em osso de baixa densidade, possibilitando uma melhor estabilidade primária, adequada para a técnica de carga imediata.

DESCRITORES | Carga Imediata; Torque; Osseointegração; Implante Dentário.

ABSTRACT | Evaluation of increased primary stability in dental implants with change in instrumentation through the integration of torque and resonance frequency analysis - Introduction: The discovery of osseointegration has set a milestone as rehabilitative therapy in dentistry, allowing for countless alternatives. Objective: The aim of this study was to evaluate an alternative technique of implant that provides a better primary stability. Materials and methods: fresh pork ribs were selected for the installation of 60 cylindrical machined implants divided into three groups. Prior to installation of the implants, bone density was measured in Hounsfield unit with conventional computed tomography. The values of bone density were measured in the position of the implants and were correlated with the insertion torque and resonance frequency analysis. In the control group, we used the installation technique in which the last drill used has $3.0 \mathrm{~mm}$, as recommended by the manufacturer, whereas in group 2 and 3 an alternative technique for sub-instrumentation and super-instrumentation was used, in which the last drill used to prepare the surgical bed had $2.8 \mathrm{~mm}$ and $3.15 \mathrm{~mm}$, respectively. The values of maximum insertion torque at the time of implant placement were recorded, and the values of resonance frequency were measured from the ratio of implant stability (ISQ). Results: An increase of $63.1219 \%$ was observed in the insertion torque when using the $2.8 \mathrm{~mm}$ as final drill, and a negative torque of insertion with $-4071 \%$ was obtained using the $3.15 \mathrm{~mm}$ drill as final drill when compared with the control group. A correlation between primary stability was verified, achieved by the average insertion torque and the diameter of the preparation of the bed receiving the implant using the cylindrical implant. Conclusion: These results indicate that by using this alternative technique for sub-instrumentation, the surgeon can reach higher values of insertion torque even at low bone density, enabling a better primary stability, suitable for the immediate loading technique.

DESCRIPTORS | Immediate Load; Torque; Osseointegration; Dental Implant.

AUTOR CORRESPONDENTE | Maurício Makoto Koga Discipline of Implantology, School of Dentistry, São Leopoldo Mandic University • Av. Reverendo José Manoel da Conceição, 796 Votorantim, SP, Brazil • 18111-000 E-mail: intrabucal@gmail.com

- Received Mar 28, 2018 • Accepted July 10, 2018

- Dol http://dx.doi.org/10.11606/issn.2357-8041.clrd.2018.144770 


\section{INTRODUÇÃO}

A descoberta da osseointegração determinou um marco para a terapia reabilitadora na odontologia, possibilitando tratamentos previsíveis. Definiu-se a osseointegração como uma conexão direta, estrutural e funcional entre o osso vivo, ordenado, e a superfície de um implante submetido à carga funcional. Esse conceito trouxe para a odontologia uma nova visão para as reabilitações até então realizadas em próteses fixas e também para a periodontia, pois a união entre osso e implantes (substitutos do elemento dental) não apresentava ligamentos periodontais, sendo semelhante à anquilose, adequando também os conceitos de oclusão e formas de distribuição das forças mastigatórias nos maxilares. ${ }^{1}$

Estudos relacionados à técnica de instalação dos implantes vêm sendo desenvolvidos para obtenção de boa estabilidade primária, principalmente nos casos onde os implantes são colocados em osso de qualidade reduzida. Tais estudos visam obter melhores resultados, mas dependem de vários fatores, como qualidade óssea, forma, modelo geométrico do implante e técnica cirúrgica. ${ }^{2}$

As alterações na superfície dos implantes acentuam a osseointegração, fato verificado por meio de análises histológicas e histomorfométricas, porém têm pouco efeito sobre a estabilidade primária. ${ }^{3}$

Há necessidade de boa estabilidade primária quando da colocação dos implantes dentais.4-7 A deficiência na estabilidade primária tem efeito significativo na perda precoce dos implantes ${ }^{8}$ quando associada à cicatrização precária. A estabilidade implantar de qualidade eficiente é essencial em osso de baixa densidade.

A ancoragem bicortical dos implantes foi sugerida como alternativa para a obtenção de uma estabilidade primária aumentada quando em osso de baixa densidade. ${ }^{8} \mathrm{O}$ preparo subdimensionado e outros procedimentos de condensação óssea foram utilizados com o propósito de aumentar a estabilidade primária. ${ }^{9}$
Implantes com diâmetro padrão $(3,75 \mathrm{~mm})$ têm sido relatados ${ }^{8}$ como os que apresentam o maior índice de falhas em osso de baixa densidade. A troca por implantes de diâmetro aumentado possibilitaria uma melhor estabilidade primária. Recomendou-se aos profissionais que fizessem uso de implantes com diâmetro mais largo onde a região da crista alveolar tivesse largura superior a $8 \mathrm{~mm}$. O uso destes implantes foi posteriormente contraindicado, pois foi relatada sua crescente perda. ${ }^{3}$

Outro grande problema também está na falta de padrão para mensurar a estabilidade primária dos implantes dentais. $^{8}$

Descreveu-se o quociente de estabilidade implantar (ISQ) como um meio útil para mensurar a estabilidade primária após a instalação dos implantes, bem como após o período de osseointegração, relatado em estudos in vitro e in vivo. A técnica mede a frequência de ressonância de um pequeno transdutor, que pode ser anexado ao implante ou a um abutment. O objetivo da técnica é avaliar a rigidez interfacial entre implante e osso e a rigidez óssea localizada no arqueamento, permitindo, desse modo, a avaliação inicial da estabilidade primária imediatamente após a colocação do implante, e possibilitando a monitorização do aumento da rigidez com a osseointegração. ${ }^{10}$

Até os dias atuais ainda não se conseguiu estabelecer um índice protocolar que determine um limite para a carga imediata com estabilidade primária alta. ${ }^{11}$

Durante certo período, o Periotest foi o método utilizado para a verificação da estabilidade primária implantar. ${ }^{12} \mathrm{O}$ Periotest envolve o uso de sonda com pequena ficha metálica que contém acelerômetro. A sonda é mantida bem próxima ao dente/dispositivo e a ficha metálica é utilizada para atingir a superfície em teste. A mobilidade é calculada a partir do tempo de contato entre a ficha metálica e a superfície do implante/dente. O Periotest é considerado sensível a alterações quanto à angulação, distância da sonda 
a partir do implante, e variação da área atingida. Foi verificado que o Periotest foi incapaz de mensurar respostas sensíveis à pequena alteração na interface implante-osso. ${ }^{12}$

A avaliação da densidade óssea é considerada um dos parâmetros importantes para prever o sucesso da terapia implantar dental a longo prazo. Embora vários tipos de classificação fossem propostos para avaliar a qualidade óssea e prever os prognósticos, um dos métodos mais populares para esse tipo de avaliação foi o proposto. ${ }^{13}$ Este classifica radiograficamente a densidade óssea em quatro tipos. Para classificar a densidade óssea de maneira objetiva e confiável, utiliza-se a tomografia computadorizada (TC) como método eficaz, também usado por vários estudos que se basearam na escala Hounsfield (HU). ${ }^{14}$

Embora a técnica de TC seja benéfica quando comparada à técnica radiográfica convencional, ela expõe o paciente a altas doses de radiação. A fim de evitar isso, recentemente foi desenvolvido o sensor cone beam, muito útil na avaliação pré-cirúrgica. A TC cone beam fornece imagem de alta resolução e não necessita da administração de altas doses de radiação, porém ainda é imprecisa quando desejamos avaliar a qualidade óssea de determinada região.

Dessa forma, a necessidade de avaliar o incremento dos valores da estabilidade primária em implantes padrão Brånemark instalados através de técnica alternativa por meio dos valores do torque de instalação e da análise de frequência de ressonância é primordial para o sucesso dos implantes em osso de baixa qualidade. Este trabalho de pesquisa buscou verificar a existência de correlação entre torque e diâmetro do preparo, mensurando o aumento da estabilidade primária por meio dos valores do torque de instalação e da análise de frequência de ressonância.

\section{MATERIAIS E MÉTODOS}

A pesquisa foi realizada nas dependências do Centro de Pesquisas Odontológicas São Leopoldo
Mandic, no Laboratório de Ensaio de Materiais. Foram obtidas 22 amostras de costelas suínas de tamanhos de diâmetro variados, tendo seus tecidos moles removidos totalmente. As costelas foram adquiridas do Frigorífico Rey Charques Ltda., situado na Rua Odorico Vieira, 267 - Sorocaba SP, registrado na Receita Federal sob o CNPJ $n^{0}$ 03.597.643/0001-52 e no Ministério da Agricultura, Pecuária e Abastecimento sob o SIF $n^{0} 1.892$. Dessas amostras, somente seis costelas foram selecionadas devido à altura óssea adequada para a instalação dos implantes, que variou de 13,23 $\mathrm{mm}$ até 16,23 $\mathrm{mm}$ (Figura 1). A razão da escolha de costelas suínas se deu devido a estas apresentarem propriedades mecânicas e de mineralização óssea semelhantes ao osso da mandíbula humana ${ }^{9}$, além disso os valores do torque de inserção mensurados nas costelas suínas são comparáveis aos do osso maxilar, sendo dessa forma um meio adequado para a verificação do aumento da estabilidade primária pela condensação óssea.

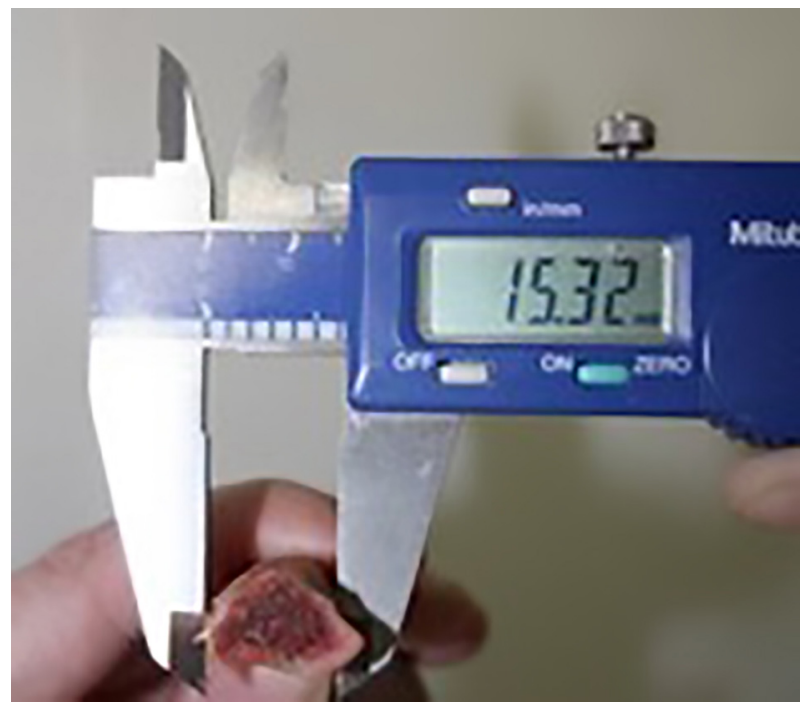

Figura 1 | Paquímetro digital conferindo a altura e a espessura das costelas suínas.

Realizou-se exame de tomografia computadorizada através do tomógrafo da marca GE CT/e Dual Slice (Figuras 2 e 3), onde as amostras foram mensuradas por meio da escala 
de medição de Hounsfield (Figura 4) para avaliar a densidade óssea. O software utilizado foi o Dental Scan, um dos programas utilizados para a obtenção de cortes axiais das tomografias odontológicas convencionais (Figura 2).

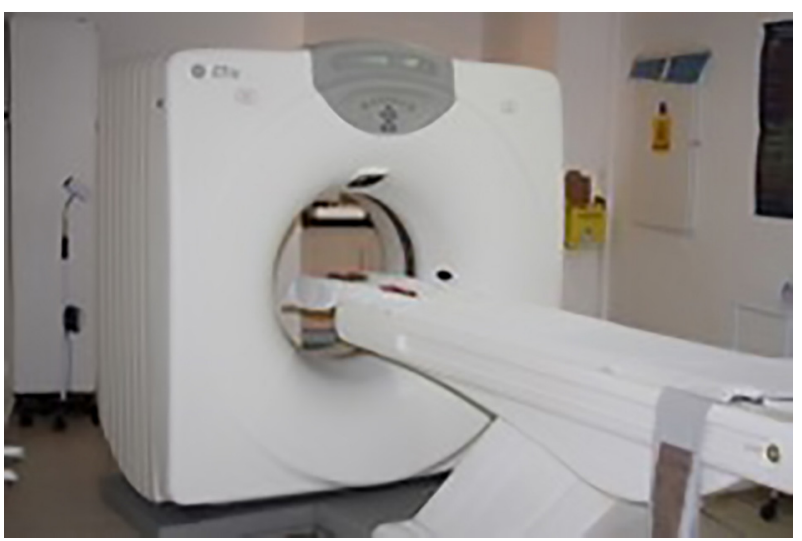

Figura 2 | Tomógrafo Ge CT/e Dual Slice.

Foram utilizados 60 implantes osseointegráveis cilíndricos com rosqueáveis de hexágono externo e plataforma regular (Conexão - ø $3,75 \mathrm{~mm} \mathrm{x}$ 11,5 mm), usinados de superfície lisa (Figura) e instalados em costelas suínas para a obtenção do objetivo de nossa pesquisa. Os implantes foram inseridos mecanicamente através do motor Koncept Surg e do contra ângulo 20:1 da marca KaVo, com torque progressivo até que dois terços do implante estivessem incluídos no osso; em seguida o implante foi inserido manualmente por meio da chave de catraca até o ponto de medição do torque. Os valores do torque de inserção máximo foram verificados através do torquímetro digital de precisão da marca Lutron TQ-880o (Figura 6), onde o extensor do montador foi adaptado ao torquímetro.

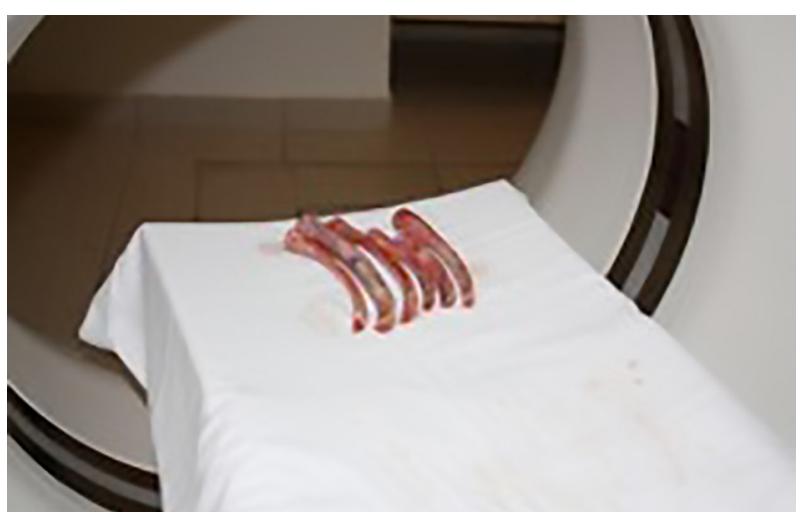

Figura 3 | Costelas suínas frescas submetidas a escaneamento pelo tomógrafo.
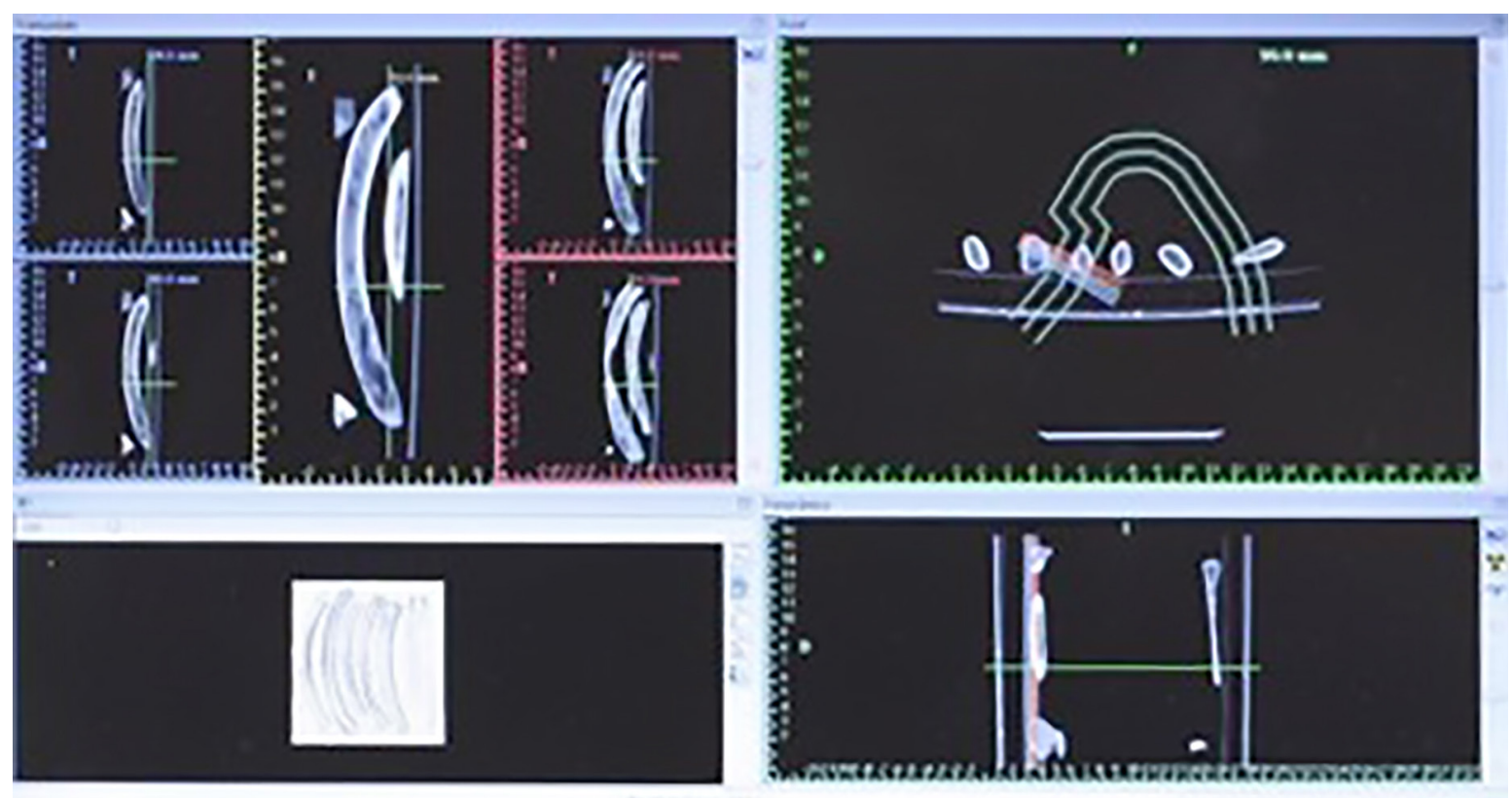

Figura 4 | Mensuração da densidade óssea em unidade Hounsfield. 


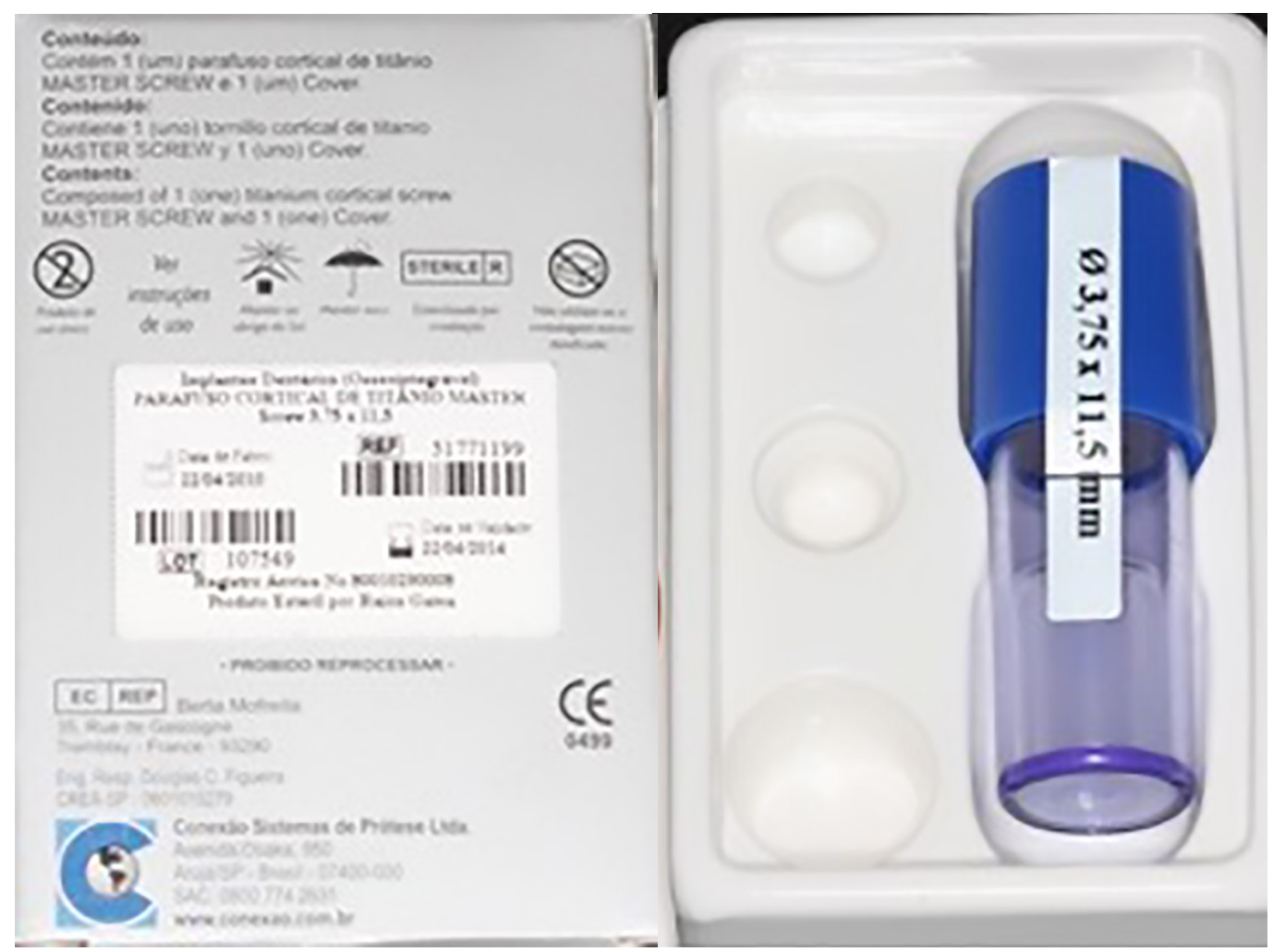

Figura 5 | Implantes cilíndricos usinados de tamanho 3,75 mm x 11,5 mm de hexágono externo com montador da marca Conexão.

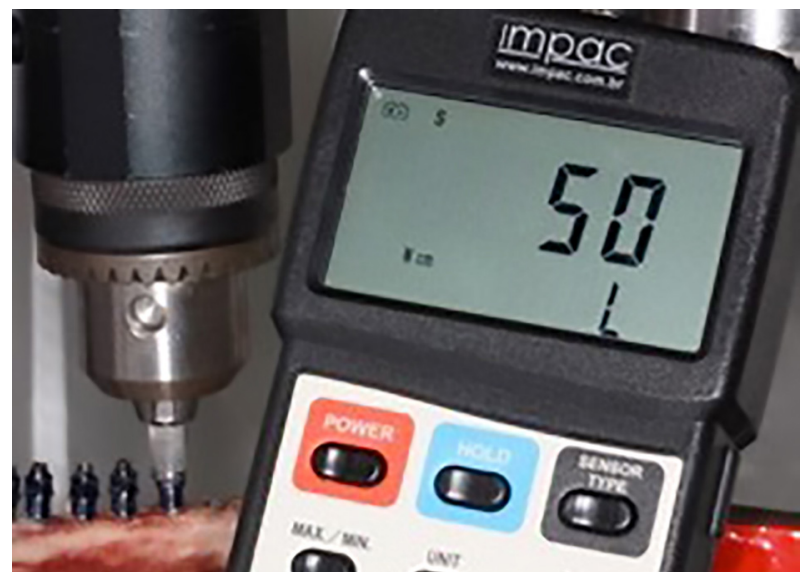

Figura 6 | Registro do valor do torque de inserção máximo obtido através do torquímetro digital.

As amostras foram divididas em três grupos, de acordo com o modo de preparo:

- grupo 1: foram instalados 20 implantes conforme a técnica preconizada pelo fabricante. Lança 2.0, piloto 2.0/3.0, 3.0 countersink;
- grupo 2: foram instalados 20 implantes tendo como broca final a de 2,8 mm. Lança 2.0, piloto 2.0/3.0, 2.8 countersink;

- grupo 3: foram instalados 20 implantes tendo como broca final a de 3,15 mm. Lança 2.0, piloto 2.0/3.0, 3.0, 3.15 countersink.

Para que não houvesse variação na manobra do operador, foi adquirida uma furadeira de bancada que foi adaptada como um suporte para imobilizar as costelas suínas (Figura 7), a fim de que não houvesse variação no longo eixo no momento do preparo do leito onde seriam fixados os implantes.

No grupo 1 a broca final foi a de $3,0 \mathrm{~mm}$ (Figura 8), e nos grupos 2 e 3 pesquisados foram utilizadas brocas de 2,8 $\mathrm{mm}$ e $3,15 \mathrm{~mm}$, respectivamente. Embora a falta de irrigação dificulte a lubrificação e a eliminação das lascas 
ósseas, isso não afetou os valores de torque de inserção durante os testes preliminares. Após instalação dos implantes e verificação do torque de inserção, foi realizada a análise de frequência de ressonância através do aparelho Osstell ISQ (Figura 9). O sistema incluiu a utilização de um SmartPeg fixado ao implante dentário através de seu parafuso. O SmartPeg é excitado por um impulso magnético da sonda de medição no instrumento portátil, e então a frequência de ressonância, que é a medição da estabilidade do implante, é calculada a partir do sinal de resposta. Os resultados são exibidos no instrumento como ISQ (Coeficiente de Estabilidade do Implante), que varia numa escala de 1 a 100. Quanto maior o número, maior a estabilidade.

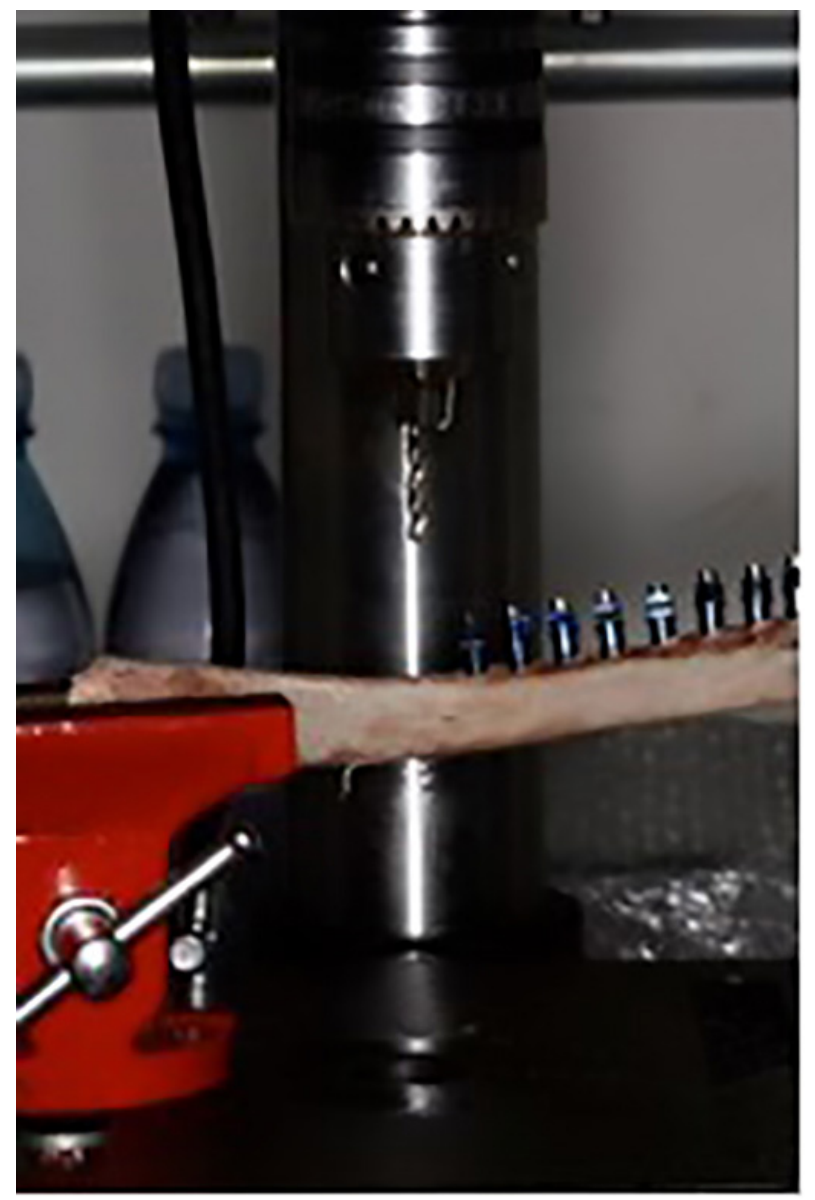

Figura 7 | Costela suína posicionada na furadeira de bancada.

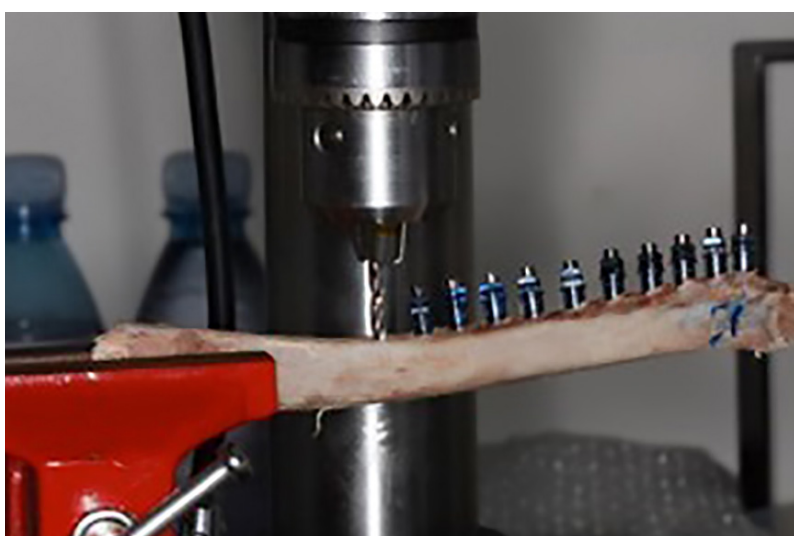

Figura 8 | Costela suína posicionada na furadeira de bancada sendo perfurada com a broca de $3,0 \mathrm{~mm}$.

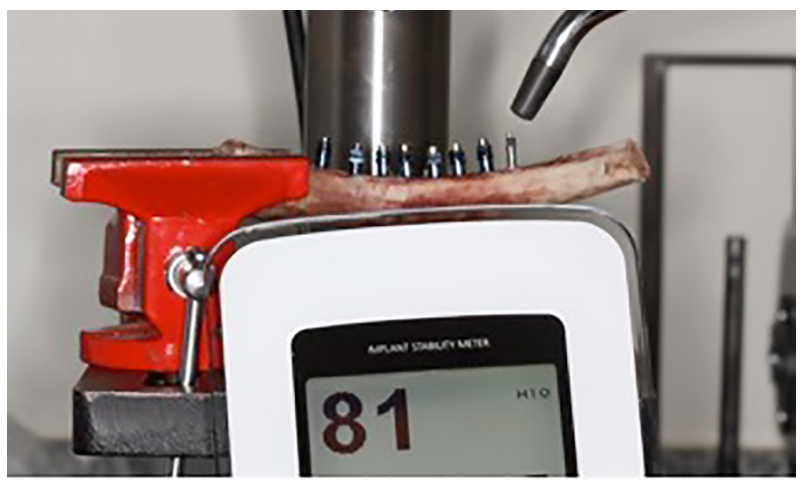

Figura 9 || Aparelho Osstell ISQ registrando o índice ISQ após instalação de implante.

\section{Resultados}

No grupo 1 (grupo-controle), foram obtidos valor médio e desvio-padrão de 142,25 \pm 25,548 HU e 24,64 $\pm 16,401 \mathrm{~N} / \mathrm{cm}$ para os valores de densidade óssea e torque, respectivamente; e valores de ISQ médio e desviopadrão de $62,00 \pm 20,230$ e 76,55 \pm 5,708 para as medições feitas perpendicularmente e no longo eixo do osso, respectivamente (Gráfico 1).

No grupo 2, foram obtidos valor médio e desviopadrão de 114,55 \pm 23,694 HU e 40,19 \pm 15,346 N/ cm para os valores de densidade óssea e torque, respectivamente; e valor de ISQ médio e desviopadrão de 62,65 \pm 19,535 e 77,80 \pm 3,172 para as medições feitas perpendicularmente e no longo eixo do osso, respectivamente (Gráfico 2). 
No grupo 3, foram obtidos valor médio e desviopadrão de 131,69 $\pm 26,471 \mathrm{HU}$ e 23,63 $\pm 11,189 \mathrm{~N} /$ $\mathrm{cm}$ para os valores de densidade óssea e torque, respectivamente; e valor de ISQ médio e desviopadrão de $67,74 \pm 10,140$ e 77,47 $\pm 3,719$ para as medições feitas perpendicularmente e no longo eixo do osso, respectivamente (Gráfico 3).

A comparação entre o grupo-controle e o grupo usando a broca de 2,8 $\mathrm{mm}$ foi muito significativa. Rejeitando-se a hipótese de nulidade e aceitando-se a alternativa, deduz-se que a broca de 2,8 $\mathrm{mm}$ foi superior à do grupo-controle em relação ao torque; no entanto, o grupo usando a broca 3,15 $\mathrm{mm}$ também foi significativo, mas com menor intensidade (Tabela 1).
Quando se comparou o ISQ obtido na medição feita no longo eixo do osso, os resultados mostraramse significantes em relação ao grupo-controle, ou seja, foram melhores que o grupo-controle (Tabela 2).

Quando se comparou o ISQ obtido na medição feita perpendicularmente ao osso, os resultados mostraram-se significantes em relação ao grupocontrole, ou seja, foram melhores que o do grupocontrole (Tabela 3).

Quando se comparou os resultados da densidade óssea dos grupos 1, 2 e 3, não houve significância estatística nos testes entre grupo-controle (1) x grupo teste $2,8 \mathrm{~mm}$ (2) e grupo-controle (1) x grupo teste 3,15 $\mathrm{mm}$ (3), devido a todos os experimentos terem utilizado os mesmos fragmentos ósseos (Tabela 4).

Gráfico 1 | Média e desvio-padrão do grupo 1.

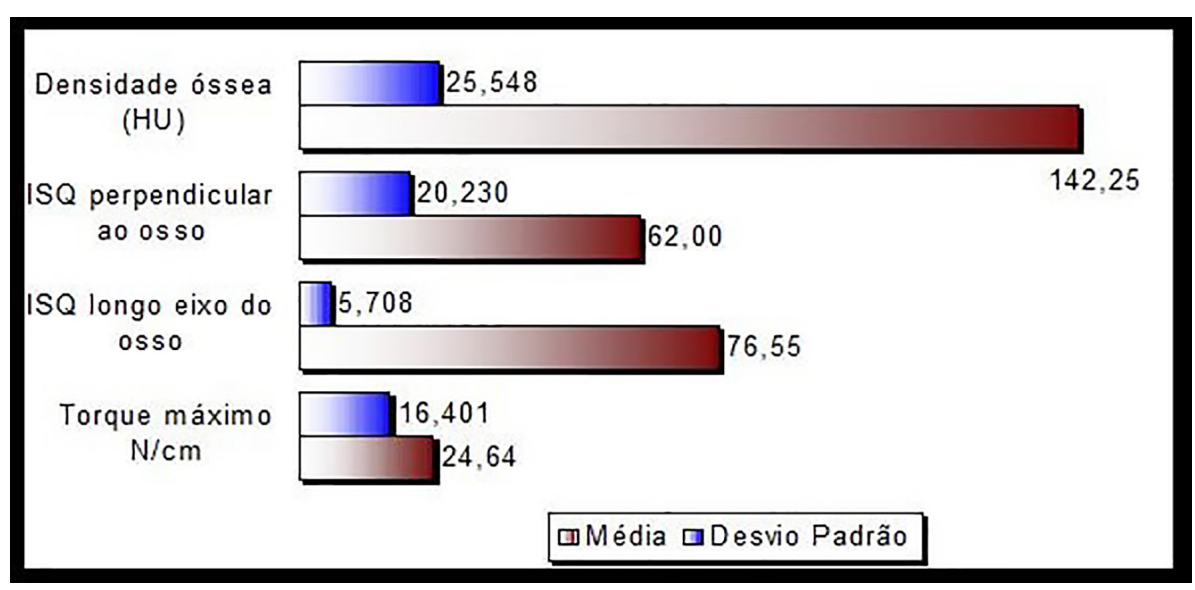

Gráfico 2 | Média e desvio-padrão do grupo 2.

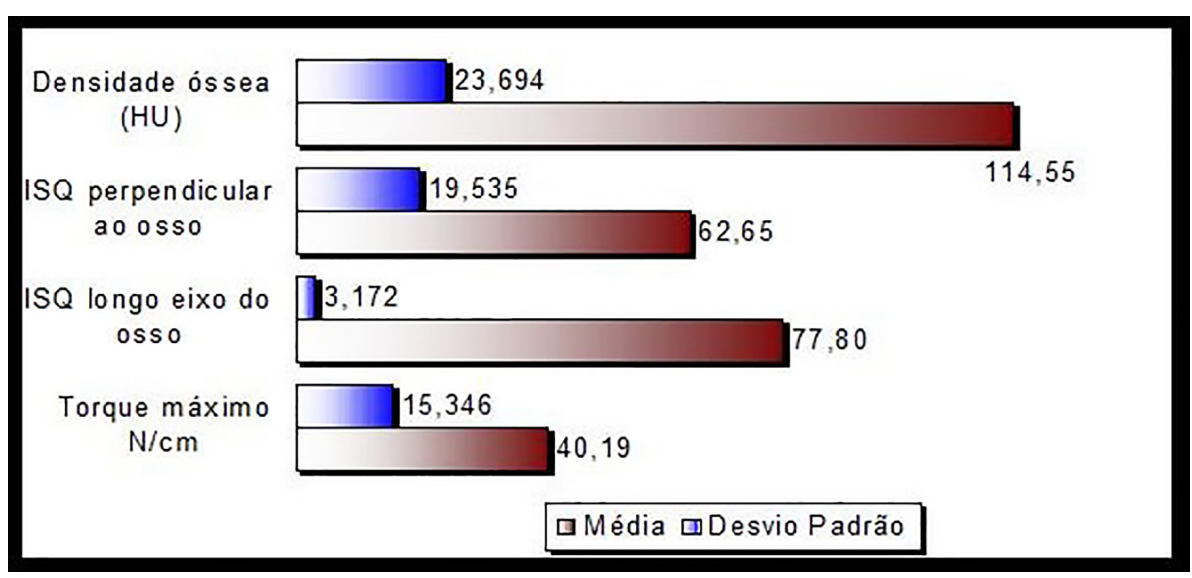


Gráfico 3 | Média e desvio-padrão do grupo 3.

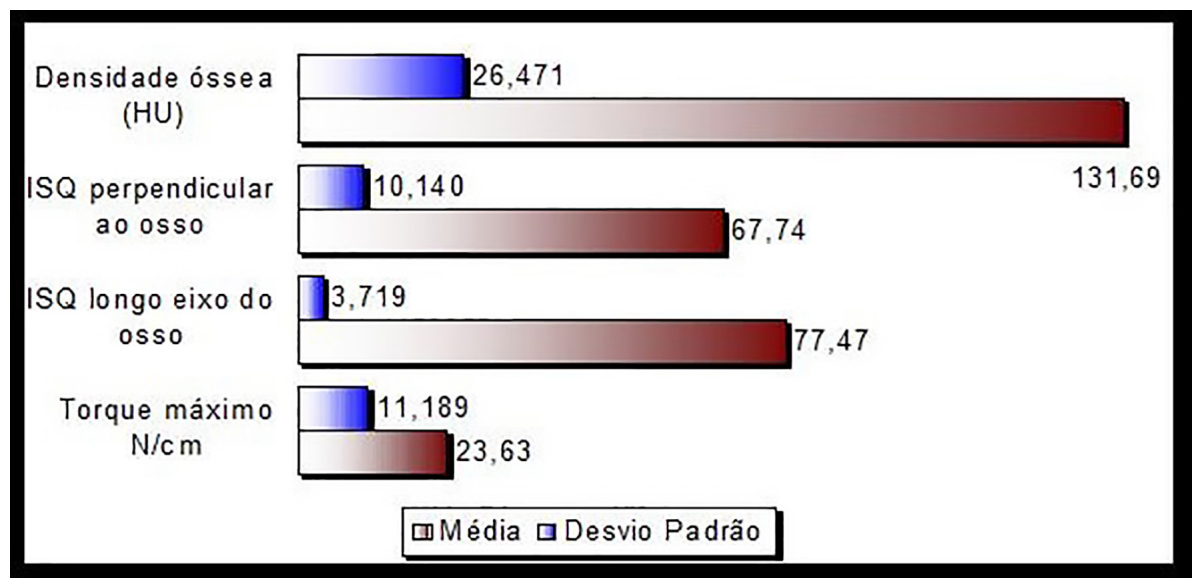

Gráfico 4 | Percentual médio de torque em relação ao grupo-controle.

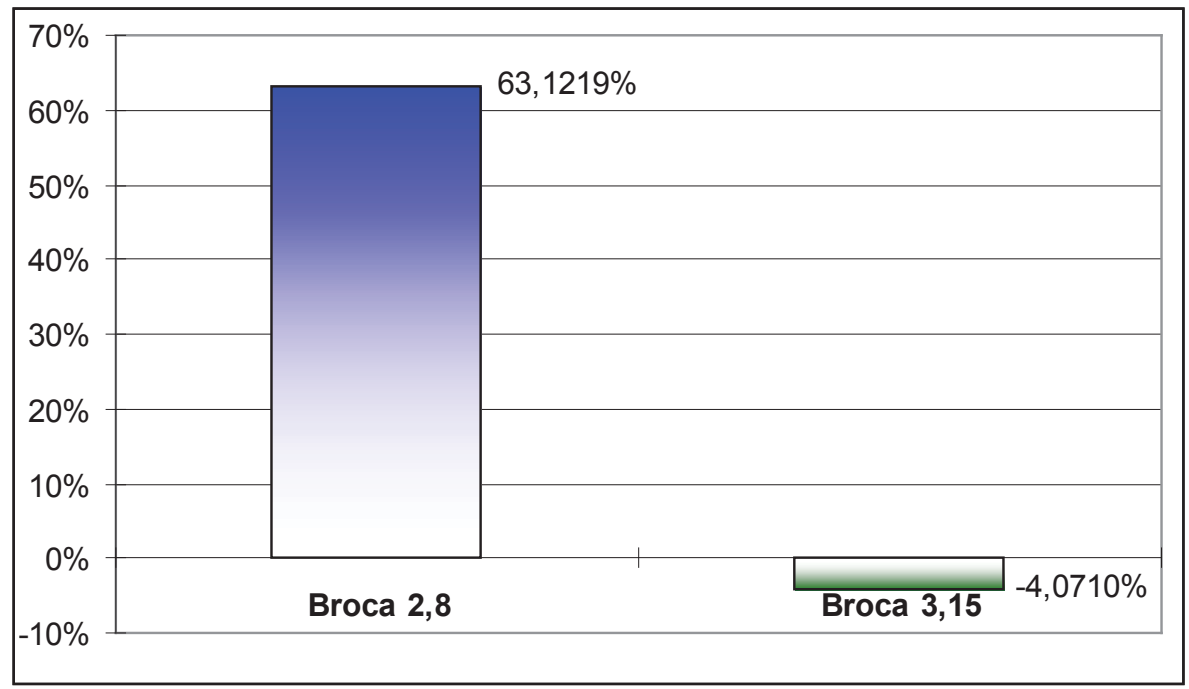

Gráfico 5 | Comparativo entre o grupo-controle e os dois testes.

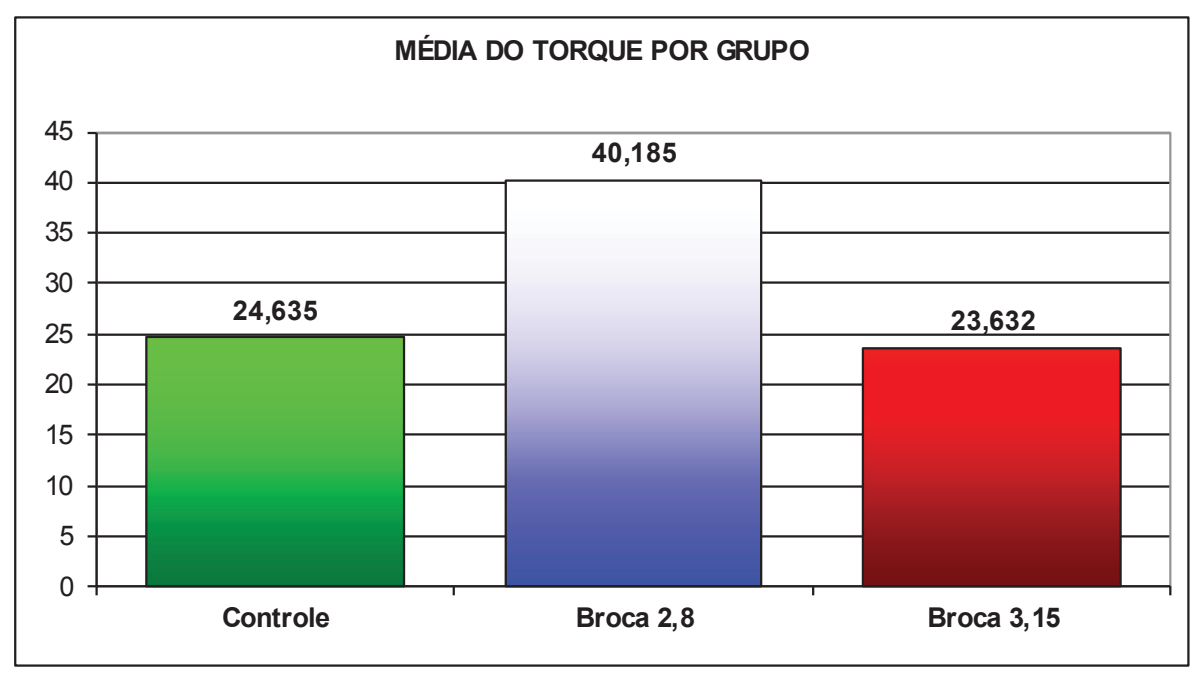


Tabela 1 | Teste t - Torque.

\begin{tabular}{|c|c|c|c|c|}
\hline \multirow[b]{2}{*}{ Indivíduos } & \multicolumn{2}{|c|}{ Controle $\mathrm{x}$ teste $(2,8 \mathrm{~mm})$} & \multicolumn{2}{|c|}{ Controle $\mathrm{x}$ teste $(3,15 \mathrm{~mm})$} \\
\hline & 20 & 20 & 20 & 20 \\
\hline Média & 24,635 & 40,14 & 24,635 & 23,63 \\
\hline Desvio-padrão & 16,4009 & 15,4002 & 16,4009 & 11,1886 \\
\hline Erro-padrão & 3,6674 & 3,4436 & 3,6674 & 2,5018 \\
\hline Desvio-padrão da diferença & 22,8704 & -- & 12,4629 & -- \\
\hline Erro-padrão da diferença & 5,114 & -- & 2,7868 & -- \\
\hline Média das diferenças & $-15,505$ & -- & 1,005 & -- \\
\hline$(t)=$ & $-3,0319 * *$ & -- & $0,3606 *$ & - \\
\hline Graus de liberdade & 19 & - & 19 & -- \\
\hline (p) unilateral = & 0,0034 & -- & 0,3612 & - \\
\hline (p) bilateral $=$ & 0,0068 & - & 0,7224 & -- \\
\hline IC (95\%) & $-26,2086$ a $-4,8014$ & -- & $-4,8277$ a 6,8377 & - \\
\hline IC (99\%) & $-30,1361$ a $-0,8739$ & & $-6,9680$ a 8,9780 & \\
\hline
\end{tabular}

*Não significante $(\mathrm{t})>0,05$

** Muito significante ao nível de (t) $<0,05$

Tabela 2 | Teste t - ISQ do longo eixo do osso.

\begin{tabular}{|c|c|c|c|c|}
\hline \multirow[b]{2}{*}{ Indivíduos } & \multicolumn{2}{|c|}{ Controle $\mathrm{x}$ teste $(2,8 \mathrm{~mm})$} & \multicolumn{2}{|c|}{ Controle $\mathrm{x}$ teste $(3,15 \mathrm{~mm})$} \\
\hline & 20 & 20 & 20 & 20 \\
\hline Média & 76,55 & 77,8 & 76,55 & 77,45 \\
\hline Desvio-padrão & 5,7076 & 3,1722 & 5,7076 & 3,7201 \\
\hline Erro-padrão & 1,2763 & 0,7093 & 1,2763 & 0,8318 \\
\hline Desvio-padrão da diferença & 6,4553 & -- & 5,1391 & -- \\
\hline Erro-padrão da diferença & 1,4435 & -- & 1,1491 & -- \\
\hline Média das diferenças & $-1,25$ & -- & $-0,9$ & -- \\
\hline$(t)=$ & $-0,866 * *$ & -- & $-0,7832 * *$ & -- \\
\hline Graus de liberdade & 19 & -- & 19 & -- \\
\hline (p) unilateral $=$ & 0,1986 & -- & 0,2216 & -- \\
\hline (p) bilateral $=$ & 0,3973 & -- & 0,4431 & -- \\
\hline IC (95\%) & $-4,2711$ a 1,7711 & -- & $-3,3052$ a 1,5052 & -- \\
\hline IC (99\%) & $-5,3797$ a 2,8797 & & $-4,1877$ a 2,3877 & \\
\hline
\end{tabular}

** Significante ao nível de (t) < 0,05. 
Tabela 3 | Teste t - ISQ perpendicular ao osso.

\begin{tabular}{|c|c|c|c|c|}
\hline \multirow[b]{2}{*}{ Indivíduos } & \multicolumn{2}{|c|}{ Controle $\mathrm{x}$ teste $(2,8 \mathrm{~mm})$} & \multicolumn{2}{|c|}{ Controle $x$ teste $(3,15 \mathrm{~mm})$} \\
\hline & 20 & 20 & 20 & 20 \\
\hline Média & 62,00 & 62,65 & 62 & 67,75 \\
\hline Desvio-padrão & 20,2303 & 19,535 & 20,2303 & 10,1405 \\
\hline Erro-padrão & 4,5236 & 4,3681 & 4,5236 & 2,2675 \\
\hline $\begin{array}{c}\text { Desvio-padrão da } \\
\text { diferença }\end{array}$ & 24,1819 & -- & 24,0676 & - \\
\hline Erro-padrão da diferença & 5,4072 & - & 5,3817 & - \\
\hline Média das diferenças & $-0,65$ & - & $-5,75$ & - \\
\hline$(\mathrm{t})=$ & $-0,1202 * *$ & - & $-1,0684 * *$ & -- \\
\hline Graus de Liberdade & 19 & - & 19 & - \\
\hline (p) unilateral $=$ & 0,4528 & -- & 0,1493 & -- \\
\hline (p) bilateral $=$ & 0,9056 & -- & 0,2987 & -- \\
\hline IC (95\%) & $-11,9674$ a 10,6674 & - & $-17,0139$ a 5,5139 & -- \\
\hline IC (99\%) & $-16,1201$ a 14,8201 & & $-21,1470$ a 9,6470 & \\
\hline
\end{tabular}

** Significante ao nível de $(\mathrm{t})<0,05$.

Tabela 4 | Teste t - Densidade óssea (HU).

\begin{tabular}{|c|c|c|c|c|}
\hline \multirow[b]{2}{*}{ Indivíduos } & \multicolumn{2}{|c|}{ Controle $\mathrm{x}$ teste $(2,8 \mathrm{~mm})$} & \multicolumn{2}{|c|}{ Controle $\mathrm{x}$ teste $(3,15 \mathrm{~mm})$} \\
\hline & 20 & 20 & 20 & 20 \\
\hline Média & 142,25 & 114,55 & 142,25 & 131,7 \\
\hline Desvio-padrão & 25,5485 & 23,6943 & 25,5485 & 26,4657 \\
\hline Erro-padrão & 5,7128 & 5,2982 & 5,7128 & 5,9179 \\
\hline $\begin{array}{l}\text { Desvio-padrão da } \\
\text { Diferença }\end{array}$ & 37,0577 & -- & 31,9069 & -- \\
\hline Erro-padrão da diferença & 8,2864 & - & 7,1346 & - \\
\hline Média das diferenças & 27,7 & - & 10,55 & - \\
\hline$(\mathrm{t})=$ & $3,3428 *$ & -- & 1,4787 * & -- \\
\hline Graus de liberdade & 19 & - & 19 & - \\
\hline (p) unilateral $=$ & 0,0017 & - & 0,0778 & - \\
\hline (p) bilateral = & 0,0034 & - & 0,1555 & - \\
\hline IC (95\%) & 10,3567 a 45,0433 & - & $-4,3827$ a 25,4827 & - \\
\hline IC (99\%) & 3,9927 a 51,4073 & & $-9,8621$ a 30,9621 & \\
\hline
\end{tabular}

*Não significante $(\mathrm{t})>0,05$ 
Tabela 5 | Correlação linear de Pearson.

\begin{tabular}{|c|c|c|c|}
\hline & Torque $\mathrm{x}$ ISQ longo & Torque $\mathrm{x}$ ISQ perpendicular & Torque $\mathrm{x}$ densidade óssea \\
\hline $\mathrm{n}($ pares $)=$ & 20 & 20 & 20 \\
\hline$r($ Pearson $)=$ & $0,2556 *$ & $0,1989 *$ & $0,0782 *$ \\
\hline $\mathrm{IC} 95 \%=$ & $-0,21$ a 0,63 & $-0,27$ a 0,59 & $-0,38$ a 0,50 \\
\hline IC 99\% = & $-0,35$ a 0,71 & $-0,40$ a 0,68 & $-0,50$ a 0,61 \\
\hline $\mathrm{R} 2=$ & 0,0653 & 0,0396 & 0,0061 \\
\hline$t=$ & 1,1218 & 0,8611 & 0,3326 \\
\hline $\mathrm{GL}=$ & 18 & 18 & 18 \\
\hline$(p)=$ & 0,2766 & 0,4005 & 0,7433 \\
\hline
\end{tabular}

*Não significante $(p)>0,05$

HO: não há correlação entre torque e outra variável $(r=0)$; H1: há correlação entre as duas variáveis $(r$ \# 0); nível de decisão: alfa = 0,05

O grupo com broca de 2,8 $\mathrm{mm}$ alcançou um torque $63,12 \%$ superior ao torque do grupocontrole; e o grupo com broca de $3,15 \mathrm{~mm}$ alcançou um torque $-4,07 \%$ inferior ao torque do grupo-controle.

O grupo com broca de 3,15 $\mathrm{mm}$ ficou com torque médio inferior ao do grupo-controle; e o grupo de broca $2,8 \mathrm{~mm}$ ficou com torque médio superior ao do grupo-controle.

Neste ensaio, a amostra extraída somente do grupo de broca 2,8 mm não apresentou correlação entre as variáveis testadas, sendo aplicada análise comparativa entre torque e ISQ longo, e entre ISQ perpendicular e densidade óssea. Os resultados foram tratados estatisticamente com o teste $t$ de Student para análise das amostras relacionadas, sempre de duas em duas- grupo 1 (controle) com outros dois (grupo 2 e grupo 3) -, alterando apenas o diâmetro da broca para os grupos, e verificada a existência de correlação entre o torque e o ISQ obtidos através do longo eixo e perpendicularmente ao osso e os valores de densidade óssea. Os resultados indicaram que existe diferença estatisticamente significativa entre um ou mais grupos analisados tanto para ISQ quanto para torque. As análises estatísticas foram realizadas para o grau de significância de $5 \%(t=0,05)$.
Os resultados médios de torque mostraram que o grupo 2 apresentou diferença estatística muito significativa em relação ao grupo 1, e não apresentou diferença estatística em relação ao grupo 3. Os valores de torque máximo determinados no grupo 3 não foram estatisticamente diferentes dos do grupo 1. Os torques de inserção do grupo 2 são diferentes dos grupos 1 e 3, uma vez que o diâmetro da osteotomia do osso é aproximadamente 6,66\% menor, proporcionando aumento de até $63,1219 \%$ no torque de inserção máximo. Aumentando o diâmetro da osteotomia para $3,15 \mathrm{~mm}$, isto é, $5 \%$, os valores do torque de inserção reduziram aproximadamente $-4,071 \%$ se comparados ao grupo 1 .

Os resultados médios de ISQ mostraram que os dados obtidos pelos grupos 2 e 3 foram estatisticamente significantes aos do grupo 1, aferidos tanto perpendicularmente quanto no longo eixo do osso. Os resultados estatísticos com relação à densidade óssea mostraram que os dados não apresentaram significância devido ao experimento ter utilizado os mesmos ossos. Quando realizamos o teste de correlação linear de Pearson comparando o torque com o ISQ obtido através das medições realizadas no longo eixo, perpendicularmente e os valores de densidade óssea, pode-se verificar a não existência de correlação entre os valores. 


\section{DISCUSSÃO}

Foi bem descrito por diversos autores em suas pesquisas a real importância da estabilidade primária na instalação dos implantes dentais. ${ }^{3-6,15,11}$ A falta de obtenção de uma eficiente estabilidade primária é condição significativa para a perda precoce dos implantes ${ }^{8}$, quando associada à precária cicatrização óssea. A estabilidade primária dos implantes na sua instalação é determinada devido à qualidade óssea, quantidade óssea, geometria das fixações e técnica de instalação dos implantes.

O tratamento da superfície dos implantes também proporcionou resultados clínicos satisfatórios na literatura recente ${ }^{16-18}$, onde foram verificadas a estabilidade primária, a densidade óssea periimplantar e o torque de remoção dos implantes. Nesse sentido, os valores do torque de inserção dos implantes são considerados importantes para avaliação de uma melhor estabilidade primária. Por essa razão, notamos o direcionamento de vários outros estudos ${ }^{19,20}$ na tentativa de avaliar melhor a presença de micromovimentações dos implantes imediatamente após sua colocação, com um aumento do torque de instalação através da análise de frequência de ressonância (RFA).

Esta pesquisa comprometeu-se a avaliar o aumento da estabilidade primária através do aumento do torque de inserção, quando da alteração da técnica de instalação preconizada pelo fabricante, bem como visualizar a correlação existente entre valores de torque e RFA. Foi registrado aumento do torque de inserção $(63,1219 \%)$ quando se utilizou a broca de $2,8 \mathrm{~mm}$ como broca final; quando se utilizou a broca de $3,15 \mathrm{~mm}$ foi obtida perda da estabilidade $(-4,071 \%)$, se comparada ao grupo-controle. Pode-se evidenciar que a variação da técnica preconizada pelo estudo é superior ao protocolo do fabricante, quando se dispõe de um osso de baixa densidade, o que proporciona uma condição favorável a possível utilização da carga imediata. ${ }^{4,11}$

Comparando ainda o torque, foi aplicado o teste $\mathrm{t}$ de Student para verificar se havia significância estatística entre os grupos, e também ficou comprovado que o grupo que utilizou a broca de $2,8 \mathrm{~mm}$, em relação ao grupocontrole, alcançou alto grau de significância $((t)=-3,0319)$, fato que não ocorreu comparandose o grupo-controle com o grupo de broca 3,15 mm, onde não se verificou significância estatística $((t)=0,3606)$. Portanto, neste estudo pode-se afirmar que o grupo de implantes dentais que utilizou a broca de $2,8 \mathrm{~mm}$ é superior aos demais em relação ao torque.

Em relação ao ISQ do longo eixo do osso, o grupo de broca 2,8 $\mathrm{mm}$ também foi superior: $1,634 \%$ contra $1,207 \%$ do grupo de broca $3,15 \mathrm{~mm}$. No ISQ perpendicular ao osso, o grupo que utilizou a broca de 3,15 $\mathrm{mm}$ alcançou média percentual 9,253\% maior que o grupo de broca $2,8 \mathrm{~mm}$, $1,049 \%$ superior à média do grupo-controle. Não houve correlação entre as variáveis testadas no teste de correlação linear de Pearson aplicado à amostra do grupo que usou a broca de $2,8 \mathrm{~mm}$, ou seja, não existe relação entre o torque e as outras variáveis testadas.

Muitas vezes pode existir variação nas medidas de RFA devido à variação do suporte ósseo coronal, influenciando na vibração do implante e reduzindo os valores de ISQ. Como essa posição foi determinada pelo pico do torque de inserção e não ao nível ósseo padrão, isso pode justificar as variações apresentadas neste estudo. ${ }^{19}$

Como resultado da pesquisa, pode-se afirmar a existência de correlação entre a estabilidade primária e o diâmetro do leito implantar com o uso de implantes cilíndricos descrito anteriormente. Dessa forma, a utilização de técnica adaptada pode proporcionar aumento do torque de inserção e melhor estabilidade primária. 
O registro da estabilidade nas diferentes fases da cicatrização dos implantes ${ }^{21}$ tem sido uma tarefa difícil de ser avaliada, sendo limitada à avaliação somente da mobilidade, o que a torna uma informação pouco precisa mesmo quando são empregados métodos mais sofisticados.

O desenvolvimento de uma técnica não invasiva eficiente para a determinação de boa estabilidade primária ocorreu quando se avaliou a frequência de ressonância no ato da instalação. Esse método revelava a ausência de mobilidade do implante instalado, e não a quantidade óssea na interface implante-osso. ${ }^{10}$

A determinação de uma boa osseointegração está diretamente relacionada à ausência de movimentação na interface osso-implante, nos diferentes tipos de densidade óssea. Portanto, a falta de micromovimentação determinada por rígida estabilidade primária e período de cicatrização isento de estímulos externos é originariamente pré-requisito para a obtenção de resultado clínico satisfatório. ${ }^{4}$

Atualmente, tem-se demonstrado em pesquisas que também é possível obter osseointegração quando os implantes são submetidos a estímulos mastigatórios imediatamente ou após sua instalação. ${ }^{11,22}$ Porém o protocolo para determinar o carregamento precoce dos implantes somente poderá ser concebido pelo alto índice de estabilidade primária inicial, com volume e densidade óssea suficientes. ${ }^{23}$ Provavelmente devido à situação crítica dos implantes carregados, ${ }^{4}$ verificou-se a importância da união de vários implantes com uma conexão rígida. Porém em seu estudo a obtenção do torque de inserção variando entre 40 e $72 \mathrm{~N} / \mathrm{cm}$ foi determinante para a carga imediata, isto posto em uma oclusão leve mesmo em ossos de pouca densidade, como o osso de qualidade tipo 4, e sem splintagem $^{24}$. Os autores verificaram os mesmos resultados satisfatórios da carga imediata quando os implantes eram instalados na maxila, porém havendo necessidade de alta estabilidade primária e colocação de implantes múltiplos suportando uma prótese fixa provisória. ${ }^{11,24}$

O sucesso da carga imediata está diretamente relacionado à estabilidade primária do implante, onde qualidade óssea, forma e modelo do implante, características da superfície do implante e técnica cirúrgica preconizada são itens importantes para sua obtenção. ${ }^{2,6,25}$ Podemos afirmar que existe correlação direta entre a RFA e a medida da estabilidade do implante através de dados histomorfométricos da ancoragem óssea; ${ }^{5}$ no entanto, para implantes instalados em osso de baixa densidade, os índices de estabilidade (RFA) no final do processo de osseointegração serão similares aos de implantes instalados em osso de densidade média e alta. Diferentemente desse resultado, não encontramos correlação entre os valores da RFA no momento da instalação dos implantes e o torque na fixação dos implantes, o que também foi relatado em outro trabalho. ${ }^{2}$ Por meio desses resultados, devemos determinar maior cautela ao conferir a análise de frequência de ressonância dos implantes dentais, isso porque limite de altura e largura dos implantes, assim como fatores de densidade óssea podem influenciar seu resultado.

Os valores do torque de inserção serem elevados devido ao preparo subdimensionado é quase um consenso na literatura, mas os valores de RFA encontrados nos diferentes estudos ${ }^{26}$ divergem com relação a sua eficácia. A perfuração subdimensionada revela o aumento do torque de inserção para ambos os tipos de implantes cônicos e cilíndricos, autorosqueantes ou não, sendo esse valor um sinal do aumento da estabilidade primária. ${ }^{6}$ Isso corrobora a teoria da falta de valor limítrofe para estabelecer o carregamento dos implantes; além disso, diferentes tipos de implantes também apresentam resultados distintos, não constituindo uma base segura para decisões terapêuticas para a carga imediata de implantes. ${ }^{8}$ 
A densidade óssea mineral tem sido discutida ${ }^{18}$ no intuito de proporcionar melhor previsibilidade no momento da instalação dos implantes, estimando a esperada estabilidade primária. Realizaram-se estudos em mandíbulas humanas empregando o uso da tomografia computadorizada (TC) como meio de ajudar o cirurgião a selecionar a melhor posição dos implantes, o tipo e a técnica operatória.9,15 Outros autores demonstraram o uso da TC como um método mais confiável para avaliar a densidade óssea, sendo que a determinação prévia da densidade óssea por tomografia computadorizada possibilita a utilização de técnica de perfuração adaptada para a obtenção de melhor estabilidade primária, como a proposta neste trabalho. ${ }^{22}$

\section{CONCLUSÃO}

Baseando-se nos resultados obtidos, é lícito concluir que:

- há correlação estatística entre a estabilidade primária obtida através do torque de inserção e o diâmetro do preparo do leito receptor do implante com a utilização de implante cilíndrico;

- a partir do uso de técnica adaptada no preparo do leito ósseo, foram obtidos valores de torque de inserção 63,1219\% maiores, se comparados aos do preparo convencional preconizado pelo fabricante, indicando ser esta técnica apropriada para casos onde o clínico disponha de osso de baixa densidade.

\section{REFERÊNCIAS}

1. Brånemark PI, Adell R, Breine U, Hansson BO, Lindström $\mathrm{J}$, Ohlsson A. Intra-osseous anchorage of dental prostheses. I. Experimental studies. Scand J Plast Reconstr Surg. 1969;3(2):81-100.

2. Javed F, Romanos GE. The role of primary stability for successful immediate loading of dental implant. A literature review. J Dent. 2010;38(8):612-20.

3. O'Sullivan D, Sennerby L, Meredith N. Measurements comparing the initial stability of five designs of dental implants: a human cadaver study. Clin Oral Implants Res. 2000;2(2):85-92.
4. Calandriello R, Tomatis M, Rangert B. Immediate functional loading of Brånemark System implants with enhanced initial stability: a prospective study 1 to 2 -year clinical and radiographic study. Clin Implant Dent Relat Res. 2003;5(Supp 1):10-20.

5. Friberg B, Jisander S, Widmark G, Lundgren A, Ivanoff C, Sennerby L, et al. One-year prospective three-center study comparing the outcome of a "soft bone implant" (prototype Mk IV) and the standard Brånemark implant. Clin Implant Dent Relat Res. 2003;5(2):71-7.

6. O’Sullivan D, Sennerby L, Jagger D, Meredith N. A comparison of two methods of enhancing implant primary stability. Clin Implant Dent Relat Res. 2004;6(1):48-57.

7. Turkyilmaz I, Tözüm TF, Tumer C, Ozbek EN. Assessment of correlation between computerized tomography values of the bone and maximum torque and resonance frequency values at dental implant placement. J Oral Rehabil. 2006;33(12):881-8.

8. Sakoh J, Wahlmann U, Stender E, Al-Nawas B, Wagner W. Primary stability of a conical implant and a hybrid, cylindric screw-type implant in vitro. Int J Oral Maxillofac Implants. 2006;21(4):560-6.

9. Beer A, Gahleitner A, Holm A, Birkfellner W, Homolka P. Adapted preparation technique for screw-type implants: explorative in vitro pilot study in a porcine bone model. Clin Oral Implants Res. 2007;18(1):103-7.

10. Meredith N, Book K, Friberg B, Jemt T, Sennerby L. Resonance frequency measurements of implant stability in vivo. A cross-sectional and longitudinal study of resonance frequency measurements on implants in the edentulous and partially dentate maxilla. Clin Oral Implants Res. 1997;8(3):226-33.

11. Östman P, Hellman M, Sennerby L. Direct implant loading in the edentulous maxilla using a bone density-adapted surgical protocol and primary implant stability criteria for inclusion. Clin Implant Dent Relat. 2005;7(Supp 1):560-9.

12. Akkocaoglu M, Uysal S, Tekdemir I, Akca K, Cehreli MC. Implant design and intraosseous stability of immediately placed implants: a human cadaver study. Clin Oral Implants Res. 2005;16(2):202-9.

13. Lekholm U, Zarb G. Introduction to osseointegration: Brånemark PI, Zarb G, George A, Albrektsson T (eds). Tissue-integrated prostheses: osseointegration in clinical dentistry. Chicago and Belin: Quitenssence; 1985. p. 11-76.

14. Schwarz MS, Rothman SL, Rhodes ML, Chafetz N. Computed tomography: part I. Preoperative assessment of the mandible for endosseous implant surgery. Int $\mathrm{J}$ of Oral Maxillofac Implants. 1987;2(3):137-41. 
15. Beer A, Gahleitner A, Holm A, Tschabitscher M, Homolka P. Correlation of insertion torques with bone mineral density from dental quantitative CT in the mandible. Clin Oral Impl Res. 2003;14(5):616-20.

16. Ferguson SJ, Langhoff JD, Voelter K, von Rechenberg B, Scharnweber D, Bierbaum S, et al. Biomechanical comparison of different surface modifications for dental implants. Int J Oral Maxillofac Implants. 2008;23(6):1037-46.

17. Kahraman S, Bal BT, Asar NV, Turkyilmaz I, Tözüm TF. Clinical study on the insertion torque and wireless resonance frequency analysis in the assessment of torque capacity and stability of self-tapping dental implants. J Oral Rehabil. 2009;36(10):755-61.

18. Trisi P, Perfetti G, Baldoni E, Berardi D, Colagiovanni M, Scogna G. Implant micromotion is related to peak insertion torque and bone density. Clin Oral Implants Res. 2009;20:467-71.

19. Akça K, Kökat AM, Cömert A, Akkocaoğlu M, Tekdemir I, Cehreli MC. Torque-fitting and resonance frequency analyses of implants in conventional sockets versus controlled bone defects in vitro. Int J Oral Maxillofac Surg. 2010;39(2):169-73.

20. Farré-Pagés N, Augé-Castro ML, Alaejos-Algarra F, Mareque-Bueno J, Ferrés-Padró E, Hernández-Alfaro F. Relation between bone density and primary implant stability. Med Oral Patol Oral Cir Bucal. 2011 Jan;16(1):e62-7.
21. Turkyilmaz I. A comparison between insertion torque and resonance frequency in the assessment of torque capacity and primary stability of Brånemark system implants. J Oral Rehabil. 2006;33(10):754-9.

22. Turkyilmaz I, Aksoy U, McGlumphy EA. Two alternative surgical techniques for enhancing primary implant stability in the posterior maxilla: a clinical study including bone density, insertion torque and resonance frequency analysis data. Clin Implant Dent Relat Res. 2008;10(4):231-7.

23. Ericsson I, Nilson H, Lindh T, Nilner K, Randow K. Immediate functional loading of Brånemark single tooth implantes. An 18 months' clinical pilot follow-up study. Clin Oral Implants Res. 2000;11(1):26-33.

24. Gapski R, Wang HL, Mascarenhas P, Lang NP. Critical review of immediate implant loading. Clin Oral Implants Res. 2003;14(5): 515-27

25. Cehreli MC, Karasoy D, Akca K, Eckert SE. Meta-analysis of methods used to assess implant stability. Int J Oral Maxillofac Implants. 2009;24(6):1015-32.

26. Rabel A, Köhler SG, Schmidt-Westhausen AM. Clinical study on the primary stability of two dental implant systems with resonance frequency analysis. Clin Oral Investig. 2007;11(3):257-65. 